\title{
Hormonal Cycle and Contraceptive Effects on Amygdala and Salience Resting-State Networks in Women with Previous Affective Side Effects on the Pill
}

\author{
Jonas Engman, ${ }^{*, 1}$, Inger Sundström Poromaa ${ }^{2}$, Lena Moby ${ }^{2}$, Johan Wikström ${ }^{3}$, Mats Fredrikson ${ }^{1,4}$ and \\ Malin Gingnell ${ }^{1,2}$ \\ 'Department of Psychology, Uppsala University, Uppsala, Sweden; 'Department of Women's and Children's Health, Uppsala University, Uppsala, \\ Sweden; ${ }^{3}$ Department of Surgical Sciences, Uppsala University, Uppsala, Sweden; ${ }^{4}$ Department of Clinical Neuroscience, Karolinska Institutet, \\ Stockholm, Sweden
}

\begin{abstract}
The mechanisms linking ovarian hormones to negative affect are poorly characterized, but important clues may come from the examination of the brain's intrinsic organization. Here, we studied the effects of both the menstrual cycle and oral contraceptives (OCs) on amygdala and salience network resting-state functional connectivity using a double-blind, randomized, and placebo-controlled design. Hormone levels, depressive symptoms, and resting-state functional connectivity were measured in 35 healthy women ( $24.9 \pm 4.2$ years) who had previously experienced $\mathrm{OC}$-related negative affect. All participants were examined in the follicular phase of a baseline cycle and in the third week of the subsequent cycle during treatment with either a combined $\mathrm{OC}$ (30 Hg ethinyl estradiol/ $0.15 \mathrm{mg}$ levonorgestrel) or placebo. The latter time point targeted the midluteal phase in placebo users and steady-state ethinyl estradiol and levonorgestrel concentrations in $O C$ users. Amygdala and salience network connectivity generally increased with both higher endogenous and synthetic hormone levels, although amygdala-parietal cortical connectivity decreased in $\mathrm{OC}$ users. When in the luteal phase, the naturally cycling placebo users demonstrated higher connectivity in both networks compared with the women receiving OCs. Our results support a causal link between the exogenous administration of synthetic hormones and amygdala and salience network connectivity. Furthermore, they suggest a similar, potentially stronger, association between the natural hormonal variations across the menstrual cycle and intrinsic network connectivity.

Neuropsychopharmacology (2018) 43, 555-563; doi:I0.1038/npp.2017.157; published online 16 August 2017
\end{abstract}

\section{INTRODUCTION}

The naturally occurring ovarian hormone fluctuations of the menstrual cycle are associated with negative affect for $~ 20 \%$ of all women in childbearing ages (Nevatte et al, 2013). This impact on well-being has been associated with the progesterone exposure in the luteal phase and is potentially intensified by the concomitantly increased levels of estradiol (Sundström Poromaa and Gingnell, 2014). Millions of women worldwide control these fluctuations with oral contraceptives (OCs) that work by creating a high synthetic hormone environment that prevents ovulation and endogenous sex hormone synthesis. However, for many, the use of OCs is also a source of negative emotional side effects (Lundin et al, 2017; Skovlund et al, 2016). This may be related to the continued exposure to progestogens in the OCs as well as the suppression of endogenous estradiol (Lundin et al, 2017; Skovlund et al, 2016).

\footnotetext{
*Correspondence: J Engman, Department of Psychology, Uppsala University, Box 1225, SE-75I 42 Uppsala, Sweden, Tel: +46 18 47| 21 07, Fax: +46 18 47I 21 23, E-mail: jonas.engman@psyk.uu.se Received 27 March 2017; revised 22 June 20 I7; accepted I 8 July 20 I7; accepted article preview online 25 July 2017
}

Little is known about the brain mechanisms mediating hormone-related negative affect, but estradiol and progesterone receptors are present in several brain areas crucial for emotion, including the amygdala and cingulate cortex (Brinton et al, 2008, 2015; Mahfouz et al, 2016; Parsons et al, 1982). Altered amygdala reactivity to emotional stimuli has been demonstrated across the menstrual cycle as well as between naturally cycling women and OC users (Petersen and Cahill, 2015; Sundström Poromaa and Gingnell, 2014). Moreover, after treatment with a gonadotropin-releasing hormone $(\mathrm{GnRH})$ agonist, downregulating estradiol levels, Henningsson et al (2015) found an association between depression symptoms and amygdala reactivity to emotional faces. An elevated anterior cingulate gyrus (ACC) density of the monoamine-regulating enzyme monoamine oxidase- $\mathrm{A}$ has been associated with symptoms of postpartum depression (Sacher et al, 2015). The dorsal part of the ACC (dACC), more specifically, has been related to hormone levels and negative affect through findings of lower dACC activity and weaker fear extinction memory in women with lower estradiol levels (Zeidan et al, 2011).

The measurement of the functional connections of the brain at rest can be used to study the brain's intrinsic 
organization into distributed networks. This is commonly examined with a seed-to-voxel approach, correlating the spontaneous low-frequency BOLD-signal oscillations in $a$ priori regions of interest (ROIs) with those in all the individual voxels of the brain. Using the amygdala as a seed defines an amygdala resting-state network (Roy et al, 2009) and the dACC the salience network (Seeley et al, 2007). The amygdala network, which includes cortical, subcortical, and cerebellar areas (Roy et al, 2009), is related to emotion processing and regulation (Vaidya and Gordon, 2013). The salience network, centered around frontal and temporal cortical and subcortical areas, is important in the detection of significant stimuli and the integration of sensory, emotional, and cognitive processes (Menon, 2015).

Alterations in both amygdala and salience resting-state functional connectivity (RSFC) have been related to negative affect (Brakowski et al, 2017), but the literature on how resting-state networks are affected by ovarian hormones and OCs is limited. Higher amygdala RSFC has been found in sensory-related areas in women with higher estradiol levels (Engman et al, 2016), as well as in prefrontal and temporal areas after estradiol infusion in postmenopausal women (Ottowitz et al, 2008a). Findings in other networks include higher hippocampus-related RSFC in women with higher estradiol and progesterone levels (Arélin et al, 2015; Lisofsky et al, 2015; Ottowitz et al, 2008b). In addition, both naturally fluctuating hormone levels and OC use have been related to alterations in the default mode and executive control networks (Petersen et al, 2014). However, mixed and negative findings have also been reported (De Bondt et al, 2015; Hjelmervik et al, 2014; Pletzer et al, 2016; Fisher et al, 2017).

In this double-blind, randomized, placebo-controlled trial, we examined how different hormonal states during the menstrual cycle and OC use affect amygdala and salience network connectivity. We hypothesized that higher hormone levels would be associated with a generally higher connectivity (Arélin et al, 2015; Engman et al, 2016; Lisofsky et al, 2015; Ottowitz et al, 2008a, 2008b). More specifically, we predicted the connectivity to be higher in frontal and sensory areas for the amygdala RSFC (Engman et al, 2016) and in frontal and temporal areas for the dACC-defined salience network RSFC (Seeley et al, 2007). The women participating in this study demonstrate increased depressive symptoms with OC use (Gingnell et al, 2013). Therefore, we also explored how change in depressive symptoms was related to change in RSFC in the women receiving OCs.

\section{MATERIALS AND METHODS}

\section{Participants}

A total of 35 healthy women $(24.9 \pm 4.2$ years $)$ with regular menstrual cycles (25 to 31 days) participated in the study. They were recruited through advertisements in local newspapers for a larger project examining the influence of OCs on brain function and negative affect. To better study this, we used an OC commonly associated with emotional side effects (Sundström Poromaa and Segebladh, 2012) and only included women who had previously suffered from OCrelated negative affect. Previous experiences of premenstrual syndrome or premenstrual dysphoric disorder were not considered. Findings from task-based paradigms collected at the same time as the resting-state data studied here have been reported elsewhere with additional details about the method (Gingnell et al, 2013, 2016). No participant used hormonal contraceptives, cortisol, levothyroxine, or psychotropic drugs for 2 months before the study and all were free from a family history of venous thromboembolism as well as neurological disorders. The presence of any ongoing psychiatric disorder was excluded using the Mini International Neuropsychiatric Interview (MINI) (Sheehan et al, 1998). Furthermore, no participant had any contraindication for MRI scanning and none was pregnant. During the study, backup contraception had to be used and no pregnancies occurred. Study procedures were in accordance with ethical standards for human experimentation and the study was approved by the Uppsala University Independent Research Ethics Committee and the Swedish Medical Products Agency (Eudra-CT: 2008-003123-124). All participants provided written informed consent before inclusion.

\section{Study Design}

Following a baseline menstrual cycle, the participants received a combined OC $(n=17)$ or placebo $(n=18)$ for one additional cycle. The OC contained $30 \mu \mathrm{g}$ ethinyl estradiol and $0.15 \mathrm{mg}$ levonorgestrel and was provided by Bayer (Berlin, Germany) who also supplied the placebo. To blind participants and study personnel from treatment, the compounds were packaged in identical capsules by Apoteksbolaget Production and Laboratories (Stockholm, Sweden). The same company performed the randomization using a computerized random-number generator in blocks of four. Group allocation was implemented by use of numbered containers. The capsules were ingested once daily, using a 21/7-day dosing regimen, starting from days 1-3 after the onset of menses. Randomization codes were kept secret at the Uppsala University Hospital Pharmacy until completion of the study. No participant reported missing a capsule and none had any left at the end of the study.

Hormone levels, depressive symptoms, and RSFC were measured in the follicular phase of the baseline cycle (day $4 \pm 3$ from the onset of menses) and during the third and final active week of the subsequent treatment cycle (day $18 \pm 2$ from the onset of treatment). The first time point was chosen to coincide with the low hormone levels of the early follicular phase for both groups. The second time point instead coincided with the high hormone levels of the midluteal phase in the naturally cycling placebo users and the steady-state ethinyl estradiol and levonorgestrel serum concentrations of the OC users. For the hormone measurements, blood samples were drawn in conjunction with the fMRI scanning sessions and immediately centrifuged and transferred to storage in $-70^{\circ} \mathrm{C}$. The serum concentrations of estradiol and progesterone were determined using a Roche Cobas e601 and Cobas Elecsys reagent kits (Roche Diagnostics, Bromma, Sweden). For estradiol, this has a detection threshold of $40 \mathrm{pmol} / \mathrm{l}$ and an intraassay coefficient of variance of $6.8 \%$ at $85.5 \mathrm{pmol} / \mathrm{l}$ and $2.8 \%$ at $1640 \mathrm{pmol} / \mathrm{l}$. For progesterone, the sensitivity is $0.1 \mathrm{nmol} / \mathrm{l}$ and the intraassay coefficient of variance $2.21 \%$ at $2.39 \mathrm{nmol} / \mathrm{l}$ and $2.82 \%$ at $31.56 \mathrm{nmol} / \mathrm{l}$. In addition, in conjunction with the fMRI scanning sessions, symptoms of depression were 
evaluated with the Montgomery-Asberg Depression Rating Scale (MADRS) (Montgomery and Asberg, 1979).

\section{Data Acquisition}

fMRI was performed with a Philips Achieva 3.0T scanner using an 8-channel head coil (Philips Medical Systems, Best, The Netherlands). Velcro strips were used to restrict head movement and earplugs to attenuate the scanner noise. During the 5-min resting-state paradigm, the participants were instructed to stay as still as possible and simply rest with their eyes closed while a single shot echo planar imaging sequence was used to collect 100 volumes of BOLD data with a voxel size of $3.0 \times 3.0 \times 3.0 \mathrm{~mm}^{3}$ in 30 ascending slices and a $\mathrm{TR}=3000 \mathrm{~ms}$, $\mathrm{TE}=35 \mathrm{~ms}$, flip angle $=90^{\circ}$, and $\mathrm{FOV}=$ $230 \times 230 \mathrm{~mm}^{2}$. For anatomical reference, an inversion recovery turbo spin echo sequence was used to acquire a structural T1-weighted image with a voxel size of $0.8 \times$ $1.0 \times 2.0 \mathrm{~mm}^{3}$ in 60 slices and $\mathrm{a} T \mathrm{TR}=5700 \mathrm{~ms}, \mathrm{TI}=400 \mathrm{~ms}$, $\mathrm{TE}=15 \mathrm{~ms}$, and $\mathrm{FOV}=230 \times 230 \mathrm{~mm}^{2}$. An influence of preceding task-based paradigms on the resting-state data cannot be excluded (Gordon et al, 2014), but is beyond the scope of this study.

\section{Preprocessing}

Scanner DICOM images were converted to nifti files with MRIcron (www.nitrc.org/projects/mricron/) and preprocessed using the DPARSF toolbox (Yan and Zang, 2010) with SPM8 (Wellcome Trust Centre for Neuroimaging). The preprocessing started with within-volume slice timing corrections and between-volume head motion corrections using six rigid body translation and rotation parameters. In the following step, the motion parameters were used as covariates of no interest together with the white matter and cerebrospinal fluid signals to reduce the sources of spurious or regionally nonspecific variance. The residual data were then spatially normalized into MNI space using DARTEL and smoothed with a $4 \mathrm{~mm}$ FWHM Gaussian kernel before a temporal band-pass filter of 0.01 to $0.08 \mathrm{~Hz}$ was applied to minimize the impact of low-frequency scanner drift and high-frequency physiological noise.

\section{Resting-State Functional Connectivity Analyses}

We examined amygdala network RSFC with AAL atlasdefined ROIs (Tzourio-Mazoyer et al, 2002) and salience network RSFC with dACC seeds defined as the AAL anterior cingulate areas posterior to the genu of the corpus callosum. These were entered into DPARSF individually, and for the left and right side separately, to calculate Pearson's product moment correlations between the mean signal time course from each seed and the time courses from all acquired voxels. In addition, Fisher's $r$-to- $z$ transformation was applied to increase the normality of the distribution of correlations.

Second-level analyses were entered directly into SPM8. Group-level amygdala and dACC RSFC was determined with one-sample $t$-tests. Differences across the cycles were examined with paired $t$-tests and differences between the groups with two-sample $t$-tests. To balance the risk of type I and type II errors, voxels were considered to be significant if they survived a cluster level FWE correction of $p<0.05$ after first having been thresholded at an uncorrected voxel level of $p<0.001$. Results were confined to gray matter with the SPM8 a priori gray matter mask binarized at a likelihood of gray matter of $>25 \%$.

\section{Demographic and Correlational Statistical Analyses}

Age, hormone level, depressive symptom, and head motion differences across the cycles were examined with paired $t$-tests, and differences between the groups with two-sample $t$-tests, using IBM SPSS Statistics 24 (IBM Corporation, Armonk, NY, USA). The association between change in depressive symptoms and change in RSFC in the OC users was examined by subtracting the follicular phase connectivity maps from those acquired during treatment, using SPM8, and correlating the resultant values with the corresponding change in MADRS scores, using bivariate correlations with Pearson's correlation coefficient in IBM SPSS Statistics 24. The analyses were confined to the areas where the OC users demonstrated a difference in RSFC across treatment.

\section{RESULTS}

\section{Demographic Data}

One naturally cycling woman receiving placebo was excluded from the follicular phase session because of excessive head movement and one discontinued the study before the session in the luteal phase. Two women receiving OCs were excluded from the treatment session because of preprocessing errors and excessive head movement. There was no difference in age between the naturally cycling placebo users $(24.3 \pm 3.3)$ and the OC users $(25.5 \pm 5.0) ; t(33)=-0.84, p=0.41$. Endogenous hormone levels did not differ in the follicular phase, but diverged between sessions (see Table 1). As expected, the naturally cycling placebo users' estradiol and progesterone serum concentrations increased, whereas the OC users' estradiol levels decreased and progesterone levels remained low. Ratings of depressive symptoms were available for 15 of the naturally cycling placebo users at each session, and for 17 and 14 of the OC users in the follicular phase and during treatment, respectively. Depressive symptoms increased in the OC users, but not among the naturally cycling placebo users, and there was no difference between the groups (see Table 1). Head motion did not exceed $1.75 \mathrm{~mm}$ translation or degrees rotation in either direction and the relative framewise displacement, as calculated by Jenkinson et al (2002) and implemented in Yan and Zang (2010), did not differ across time or between the groups (all $p s>0.10$ ). In an additional step to better ascertain that head motion did not affect our analyses, we performed a diagnostic test in which the participant with the relatively most between-volume movement was removed from one of the contrasts. The impact on the result was negligible.

\section{Amygdala and Salience Network Connectivity}

In the follicular phase, amygdala and dACC RSFC covered widespread areas of the brain across all participants combined (see Supplementary Figure S1). The amygdala network largely corresponded to that demonstrated in 
Table I Hormone Levels and Depressive Symptoms

\begin{tabular}{|c|c|c|c|c|c|c|c|c|}
\hline & \multicolumn{3}{|c|}{ Naturally cycling placebo users } & \multicolumn{3}{|c|}{ Oral contraceptive users } & \multicolumn{2}{|c|}{ Between-group effects } \\
\hline & $\begin{array}{l}\text { Follicular } \\
\text { phase }\end{array}$ & $\begin{array}{l}\text { Luteal } \\
\text { phase }\end{array}$ & $\begin{array}{l}\text { Within-group } \\
\text { effects }(p)\end{array}$ & $\begin{array}{l}\text { Follicular } \\
\text { phase }\end{array}$ & $\begin{array}{c}\text { During } \\
\text { treatment }\end{array}$ & $\begin{array}{l}\text { Within-group } \\
\text { effects }(p)\end{array}$ & $\begin{array}{l}\text { Follicular } \\
\text { phase }(p)\end{array}$ & $\begin{array}{c}\text { Luteal phase/During } \\
\text { treatment }(p)\end{array}$ \\
\hline Estradiol $(\mathrm{pmol} / \mathrm{l})^{\mathrm{a}}$ & $167 \pm 99$ & $436 \pm 277$ & 0.002 & $243 \pm 175$ & $50 \pm 18$ & 0.001 & 0.13 & $<0.001$ \\
\hline Progesterone $(\mathrm{nmol} / \mathrm{l})$ & $2.0 \pm 0.9$ & $18.2 \pm 21.2$ & 0.006 & $3.1 \pm 4.2$ & $1.4 \pm 0.5$ & 0.16 & 0.30 & 0.004 \\
\hline
\end{tabular}

Hormone levels and symptom scores are presented as mean $\pm \mathrm{SD}$, and the significance levels for the within- and between-group effects are given in $p$-values.

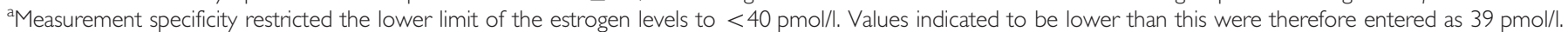

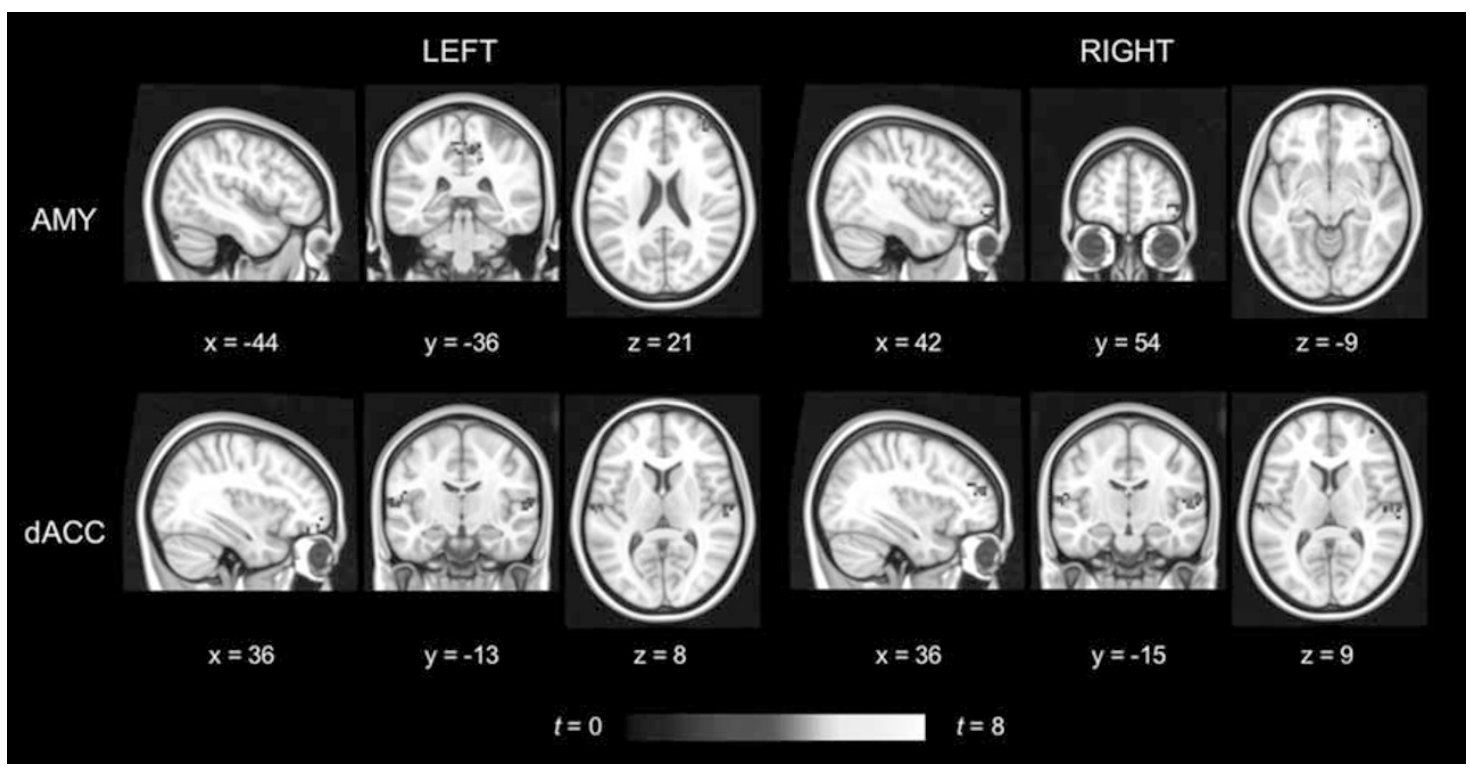

Figure I Within-group differences for naturally cycling placebo users. The hot color scale indicates areas with increased resting-state functional connectivity in the left and right amygdala (AMY) and dorsal anterior cingulate cortex (dACC) respectively in the luteal compared with the follicular phase. No area with higher connectivity in the follicular phase was found. Coordinates are given in $\mathrm{MNI}$ space and statistics in $t$-values. A full color version of this figure is available at the Neuropsychopharmacology journal online.

previous studies (Gabard-Durnam et al, 2014; Roy et al, 2009) as did the network defined by the dACC seeds to the salience network (Menon, 2015; Seeley et al, 2007).

\section{Within- and Between-Group Differences}

The naturally cycling placebo users demonstrated no areas with higher RSFC in the follicular compared with the luteal phase, but a widespread increase in RSFC in the luteal compared with the follicular phase (see Figure 1 and Table 2). This included higher amygdala RSFC to the middle and superior frontal gyri, paracentral lobule, and cerebellum. At the same time, dACC RSFC was higher in the middle frontal, superior temporal, transverse temporal, and postcentral gyri.

The OC users demonstrated higher dACC RSFC in the superior frontal gyrus and precuneus during treatment compared with the follicular phase, whereas their amygdala RSFC was higher in the postcentral gyrus in the follicular phase compared with that during treatment (see Figure 2 and Table 2).

The two groups demonstrated no differences in amygdala and salience network RSFC in the follicular phase. However, during treatment, the naturally cycling placebo users had higher amygdala RSFC in the postcentral gyrus and cuneus, as well as higher dACC RSFC in the precuneus, than the OC users (see Figure 3 and Table 2).

The mean connectivity values in the peak voxels within the significant clusters from the within- and between-group analyses, for the separate sessions and groups respectively, were positive with a few exceptions. In the group of naturally cycling placebo users, the mean amygdala connectivity values in the middle and superior frontal gyri, as well as in the cerebellum, were negative in the follicular phase. This was also true of their left dACC RSFC in the middle frontal gyrus.

\section{Correlations}

In the areas where the OC users demonstrated altered connectivity across the treatment, no correlations were found between change in RSFC and change in depressive symptoms.

\section{DISCUSSION}

In this randomized, double-blind, placebo-controlled trial, we examined amygdala and dACC RSFC in women receiving a combined OC or placebo. Across all participants in the 
Table 2 Within- and Between-Group Differences in Amygdala and Dorsal Anterior Cingulate Cortex (dACC) Resting-State Functional Connectivity for Naturally Cycling Placebo Users and Oral Contraceptive Users

\begin{tabular}{|c|c|c|c|c|c|c|}
\hline & BA & $x$ & $y$ & $\mathbf{z}$ & z-Score & Cluster size \\
\hline \multicolumn{7}{|c|}{ Within-group differences for naturally cycling placebo users } \\
\hline \multicolumn{7}{|c|}{ Luteal phase $>$ baseline follicular phase } \\
\hline \multicolumn{7}{|l|}{ Left amygdala } \\
\hline Cerebellum & & -48 & -75 & -36 & 4.28 & 35 \\
\hline Paracentral lobule & 5 & -3 & -36 & 51 & 4.00 & 123 \\
\hline Superior frontal gyrus & 10 & 36 & 60 & 21 & 3.73 & 34 \\
\hline \multicolumn{7}{|l|}{ Right amygdala } \\
\hline Middle frontal gyrus & & 42 & 54 & -9 & 4.57 & 37 \\
\hline \multicolumn{7}{|l|}{ Left dACC } \\
\hline Middle frontal gyrus & & 36 & 51 & -15 & 4.36 & 49 \\
\hline Superior temporal gyrus & & 60 & -15 & 6 & 4.07 & 31 \\
\hline Transverse temporal gyrus & 41 & -51 & -27 & 12 & 3.81 & 54 \\
\hline \multicolumn{7}{|l|}{ Right dACC } \\
\hline Middle frontal gyrus & 10 & 36 & 48 & 15 & 3.90 & 41 \\
\hline Transverse temporal gyrus & 42 & 60 & -15 & 9 & 3.87 & 49 \\
\hline Postcentral gyrus & 43 & -54 & -15 & 15 & 3.62 & 29 \\
\hline \multicolumn{7}{|c|}{ Within-group differences for oral contraceptive users } \\
\hline \multicolumn{7}{|c|}{ Baseline follicular phase $>$ during treatment } \\
\hline \multicolumn{7}{|l|}{ Right amygdala } \\
\hline Postcentral gyrus & 40 & -51 & -36 & 54 & 4.03 & 28 \\
\hline \multicolumn{7}{|c|}{ During treatment $>$ baseline follicular phase } \\
\hline \multicolumn{7}{|l|}{ Left dACC } \\
\hline Precuneus & & 0 & -63 & 33 & 4.82 & 70 \\
\hline \multicolumn{7}{|l|}{ Right dACC } \\
\hline Precuneus & 7 & -3 & -69 & 33 & 4.87 & 55 \\
\hline Superior frontal gyrus & 10 & -3 & 66 & 18 & 4.66 & 38 \\
\hline \multicolumn{7}{|l|}{ Between-group differences } \\
\hline \multicolumn{7}{|c|}{ Naturally cycling placebo users in the luteal phase $>O C$ users during treatment } \\
\hline \multicolumn{7}{|l|}{ Left amygdala } \\
\hline Cuneus & 18 & -18 & -81 & 21 & 4.38 & 35 \\
\hline Postcentral gyrus & 3 & -54 & -21 & 42 & 4.34 & 42 \\
\hline \multicolumn{7}{|l|}{ Left dACC } \\
\hline Precuneus & & 0 & -54 & 63 & 3.92 & 53 \\
\hline
\end{tabular}

All clusters are significant at $p<0.05$ family-wise error (FWE) corrected for multiple comparisons. Secondary local maxima within the significant clusters are not listed.

follicular phase combined, the resultant networks corresponded well to previously published descriptions (GabardDurnam et al, 2014; Menon, 2015; Roy et al, 2009; Seeley et al, 2007). In line with our hypotheses, we generally found higher connectivity with higher hormone levels. This was reflected in increased amygdala and dACC RSFC in the luteal compared with the follicular phase in women receiving placebo, as well as increased dACC RSFC during treatment compared with the follicular phase in OC users. In addition, in line with our hypotheses, clusters with higher amygdala RSFC were predominantly found in the frontal cortex and sensory areas, whereas clusters with higher dACC RSFC were primarily located not only in prefrontal and temporal areas, but also in parietal areas. A contrasting finding was demonstrated in amygdala-inferior parietal RSFC that decreased with OC use. When the groups were compared during treatment, the naturally cycling placebo users demonstrated higher amygdala and dACC RSFC than the OC users. Taken together, this indicates similarities in the effects of endogenous and exogenous hormone levels on the resting-state networks, but suggests that the effect of endogenous hormones is somewhat more profound. The exception to higher hormone levels being associated with higher intrinsic connections in the brain-the reduced amygdala-parietal connectivity as a function of OC use-may reflect a reduced coupling between the amygdala and cortical areas consistent 


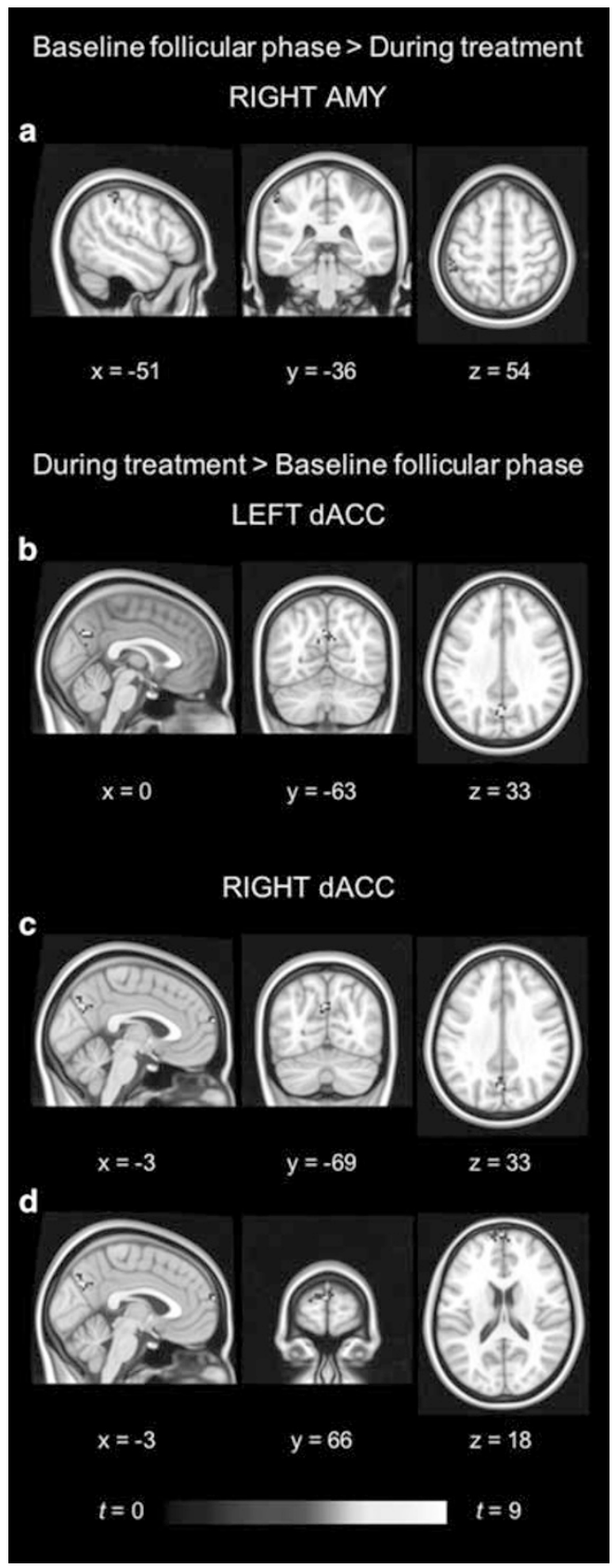

Figure 2 Within-group differences for oral contraceptive users. The hot color scale indicates (a) higher right amygdala-postcentral gyrus resting-state functional connectivity (RSFC) in the follicular phase than during treatment, as well as increased (b) left dorsal anterior cingulate cortex (dACC)-precuneus, (c) right $\mathrm{dACC}$-precuneus, and (d) right $\mathrm{dACC}$-superior frontal gyrus RSFC during treatment compared with the follicular phase. Coordinates are given in $\mathrm{MNI}$ space and statistics in t-values. A full color version of this figure is available at the Neuropsychopharmacology journal online.

with a reduced relation between emotion and cognition during OC use. No correlations were found between the OC users' change in depressive symptoms and RSFC across the treatment.

\section{Hormones and RSFC}

Our results are consistent with reports of an association between hormonal state and RSFC (Arélin et al, 2015;

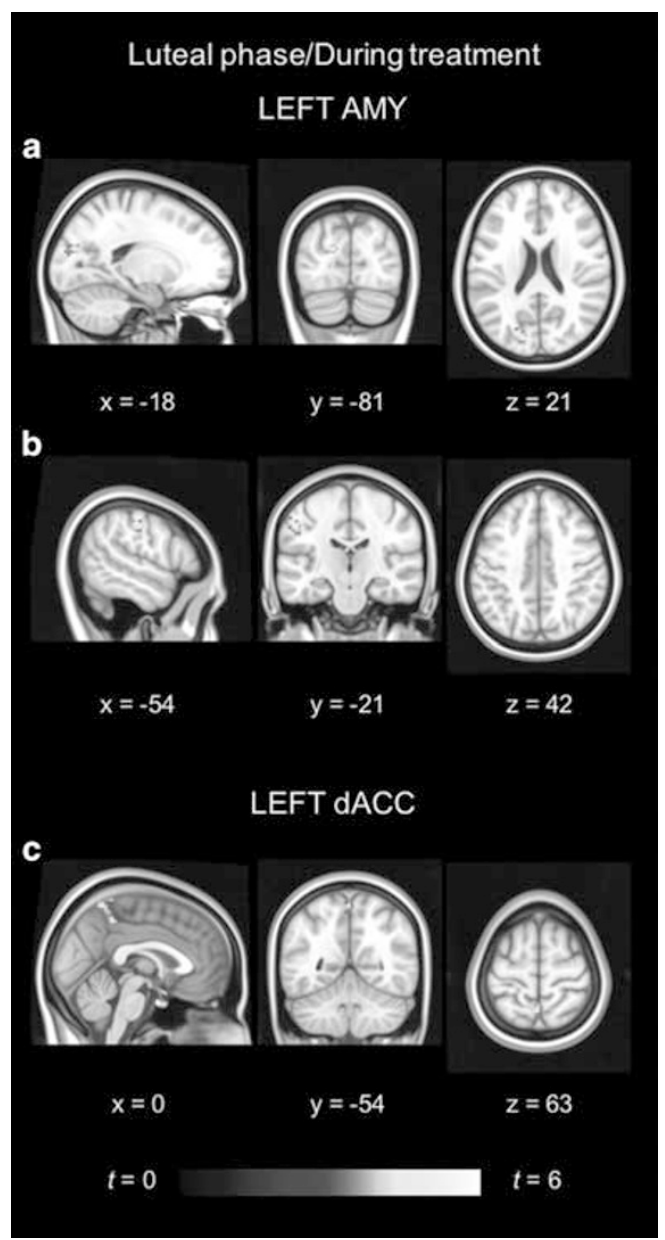

Figure 3 Between-group differences. The hot color scale indicates higher resting-state functional connectivity between the (a) left amygdala and cuneus, (b) left amygdala and postcentral gyrus, as well as the (c) left dorsal anterior cingulate cortex (dACC) and precuneus in the naturally cycling placebo users compared with the oral contraceptive users during treatment. No area with higher connectivity for the oral contraceptive users during treatment, or for either group in the follicular phase, was found. Coordinates are given in $\mathrm{MNI}$ space and statistics in $t$-values. A full color version of this figure is available at the Neuropsychopharmacology journal online.

Engman et al, 2016; Lisofsky et al, 2015; Ottowitz et al, 2008a, 2008b; Petersen et al, 2014). Similar to the findings in the naturally cycling placebo users, two previous studies demonstrate generally higher amygdala RSFC with higher estradiol levels (Engman et al, 2016; Ottowitz et al, 2008a), although another found no main effect of hormones, and only a marginally mediating effect of amygdala RSFC on the association between estradiol and depressive symptoms in women receiving a GnRH agonist (Fisher et al, 2017). In contrast, effects of naturally cycling hormones and OCs on default mode and cognitive control networks (Petersen et al, 2014) have resulted in mixed (Pletzer et al, 2016) and null findings (De Bondt et al, 2015; Hjelmervik et al, 2014). One potential explanation for these inconsistencies may lie in the functions supported by the different networks and how they are affected by fluctuations in hormone levels (Pletzer et al, 2016). The default mode and executive control networks are mainly related to cognitive functions whereas the amygdala network is associated with emotional processes (Vaidya and Gordon, 2013), and a recent review found menstrual cycle 
effects to be small on cognitive functions, but more commonplace on emotion-related processes (Sundström Poromaa and Gingnell, 2014). The alterations in salience network connectivity among the naturally cycling placebo users in this study, and reported previously for hippocampal RSFC (Arélin et al, 2015; Lisofsky et al, 2015), may also suggest that, although these networks support both cognitive and emotional processes (Vaidya and Gordon, 2013), associations to emotional functions predominate in studies producing consistent results. The progesteronedominated luteal phase also comes with many bodily changes that the salience network is well equipped to detect, whereas the hippocampal formation has been associated with verbal memory (Sundström Poromaa and Gingnell, 2014), a cognitive function that does appear to be linked to hormones (Nolte, 2009).

\section{Amygdala RSFC}

In the luteal phase, the naturally cycling placebo users demonstrated increased amygdala RSFC in the middle and superior frontal gyri, paracentral lobule, and cerebellum. The paracentral lobule and cerebellum are associated with motor and sensory processes (Koziol et al, 2011; Nolte, 2009). The latter has also been related to emotional processes (Sang et al, 2012). These changes may therefore suggest an increased sensitivity in perception and emotionally determined processes. The middle and superior frontal gyri have been implicated in emotional regulation (Frank et al, 2014). The connectivity to these areas in the naturally cycling placebo users was right-lateralized, negative in the follicular phase and positive in the luteal phase. We speculate that this pattern of results may reflect a mechanism involved in emotion regulation.

In contrast to our hypothesis, the OC users had lower amygdala RSFC in the postcentral gyrus during treatment compared with before. During treatment, their amygdala connectivity to the postcentral gyrus, as well as to the cuneus, was also lower than that of the naturally cycling placebo users, indicating reduced subcortical-cortical connections. This may be explained by the fact that the progestogen levonorgestrel in our OC is a testosterone derivative with affinity for progesterone as well as androgen and mineralocorticoid receptors (Hapgood et al, 2014). That opens for androgenic actions that may deviate from the endocrine environment that naturally cycling women are exposed to in the luteal phase and potentially counteract estradiol-induced connectivity. In line with this, testosterone has been suggested to decrease subcortical-cortical connectivity (Peper et al, 2011).

\section{Salience Network RSFC}

In the luteal phase, the naturally cycling placebo users demonstrated increased dACC RSFC in the postcentral, superior temporal, and transverse temporal gyri, which are all related to sensory processes (Nolte, 2009). This increased sensitivity within the salience network during the luteal phase may reflect increased vigilance and awareness of bodily sensations. Furthermore, the increased connectivity between emotion-related and cortical areas within the salience network are consistent with an increased emotionality related to a reduced cognitive control in the luteal phase.

In contrast to the increased positive RSFC in the OC users, healthy controls have demonstrated a negative connection between the dACC and superior frontal gyrus (Zhang et al, 2015). This change also coincided with increased depressive symptoms, suggesting it may play a role in negative mood, although we found no linear correlation between the two.

\section{Limitations and Future Studies}

Some important limitations need to be considered. We only included women who had previously experienced negative mood side effects from OCs, and therefore our study may not generalize to all women. These prior experiences may also have affected the participants' expectations and biased the results. However, that the same proportion of women in each group guessed their treatment correctly argues against this (Gingnell et al, 2013). Furthermore, the scanning sessions in the naturally cycling placebo users were scheduled based on an idealized 28-day menstrual cycle. An individual monitoring of hormone levels would have made it possible to more securely place these women in the luteal phase at the second scanning session. However, this could have compromised the blinding of the study and introduced systematic differences between the groups. Even though the use of a double-blind, randomized, controlled trial design and the longitudinal approach constitute significant strengths of the study, the low hormone session preceded the high hormone session for all participants, making it difficult to evaluate potential session effects. However, RSFC has proven reliable in previous studies (see, eg, Guo et al, 2012). It should also be noted that the final sample size was relatively modest for the between-group comparisons. Finally, there are factors potentially affecting RSFC patterns that we did not control for, including respiration and pulse (Craddock et al, 2013).

The neurobiological mechanism through which ovarian steroid hormones influence resting-state network connectivity remains to be disentangled. It may include a direct effect of estradiol and progesterone on nervous system excitability or synchronicity as sex steroid hormone receptors are abundant throughout the brain (Mahfouz et al, 2016) as well as the modulation of neurotransmission. The implication of the serotonergic system in the regulation of negative affect and its treatment (Olivier, 2015) would make it particularly interesting in the context of this study, and estradiol and progesterone administration has, eg, been shown to increase whole brain expression of the serotonin 2 A receptor (Moses et al, 2000). Both neural excitability and neurochemical mediators are thus candidates for further examination to elucidate the mechanism mediating hormonal effects on brain activity and connectivity.

\section{CONCLUSIONS}

In this double-blind, randomized, placebo-controlled trial, we found that both endogenous and exogenous hormone levels, linked to the menstrual cycle and OCs respectively, influenced amygdala and salience resting-state networks, 
with generally higher RSFC with higher hormone levels. The connectivity patterns support an increased sensitivity in both networks and suggest a potential protective mechanism for emotion regulation.

\section{FUNDING AND DISCLOSURE}

ISP occasionally serves on advisory boards or is invited to give talks at scientific meetings for MSD, Bayer Health Care, and Lundbeck A/S. The other authors declare no conflict of interest. This study was funded by the Swedish Research Council (2016-01439), the Swedish Council for Working Life and Social Research (2007-1955 and 2007-2116), as well as by the Family Planning Foundation. It was also supported in part by an unrestricted research grant from Bayer $A B$, but the authors alone are responsible for the content and writing of the manuscript.

\section{ACKNOWLEDGMENTS}

The amygdala resting-state functional connectivity data were presented in a short oral presentation at the 32nd Annual Scientific Meeting of the European Society for Magnetic Resonance in Medicine and Biology, Edinburgh, UK, and the salience network resting-state functional connectivity data as a poster at the 22nd Annual Meeting of the Organization for Human Brain Mapping, Geneva, Switzerland.

\section{REFERENCES}

Arélin K, Mueller K, Barth C, Rekkas PV, Kratzsch J, Burmann I et al (2015). Progesterone mediates brain functional connectivity changes during the menstrual cycle-a pilot resting-state MRI study. Front Neurosci 9: 44.

Brakowski J, Spinelli S, Dörig N, Bosch OG, Manoliu A, Holtforth MG et al (2017). Resting state brain network function in major depression - depression symptomatology, antidepressant treatment effects, future research. J Psychiatr Res 92: 147-159.

Brinton RD, Thompson RF, Foy MR, Baudry M, Wang J, Finch CE et al (2008). Progesterone receptors: form and function in brain. Front Neuroendocrinol 29: 313-339.

Brinton RD, Yao J, Yin F, Mack WJ, Cadenas E (2015). Perimenopause as a neurological transition state. Nat Rev Endocrinol 11: 393-405.

Craddock RC, Jbabdi S, Yan C-G, Vogelstein JT, Castellanos FX, Di Martino A et al (2013). Imaging human connectomes at the macroscale. Nat Methods 10: 524-539.

De Bondt T, Smeets D, Pullens P, Van Hecke W, Jacquemyn Y, Parizel PM (2015). Stability of resting state networks in the female brain during hormonal changes and their relation to premenstrual symptoms. Brain Res 1624: 275-285.

Engman J, Linnman C, Van Dijk KRA, Milad MR (2016). Amygdala subnuclei resting-state functional connectivity sex and estrogen differences. Psychoneuroendocrinology 63: 34-42.

Fisher PM, Larsen CB, Beliveau V, Henningsson S, Pinborg A, Holst KK et al (2017). Pharmacologically induced sex hormone fluctuation effects on resting-state functional connectivity in a risk model for depression: a randomized trial. Neuropsychopharmacology 42: 446-453.

Frank DW, Dewitt M, Hudgens-Haney M, Schaeffer DJ, Ball BH, Schwarz NF et al (2014). Emotion regulation: quantitative meta- analysis of functional activation and deactivation. Neurosci Biobehav Rev 45: 202-211.

Gabard-Durnam LJ, Flannery J, Goff B, Gee DG, Humphreys KL, Telzer E et al (2014). The development of human amygdala functional connectivity at rest from 4 to 23 years: a crosssectional study. Neuroimage 95: 193-207.

Gingnell M, Bannbers E, Engman J, Frick A, Moby L, Wikström J et al (2016). The effect of combined hormonal contraceptives use on brain reactivity during response inhibition. Eur J Contracept Reprod Health Care 21: 150-157.

Gingnell M, Engman J, Frick A, Moby L, Wikström J, Fredrikson M et al (2013). Oral contraceptive use changes brain activity and mood in women with previous negative affect on the pill-A double-blinded, placebo-controlled randomized trial of a levonorgestrel-containing combined oral contraceptive. Psychoneuroendocrinology 38: 1133-1144.

Gordon EM, Breeden AL, Bean SE, Vaidya CJ (2014). Working memory-related changes in functional connectivity persist beyond task disengagement. Hum Brain Mapp 35: 1004-1017.

Guo CC, Kurth F, Zhou J, Mayer EA, Eickhoff SB, Kramer JH et al (2012). One-year test-retest reliability of intrinsic connectivity network fMRI in older adults. Neuroimage 61: 1471-1483.

Hapgood JP, Africander D, Louw R, Ray RM, Rohwer JM (2014). Potency of progestogens used in hormonal therapy: toward understanding differential actions. J Steroid Biochem Mol Biol 142: 39-47.

Henningsson S, Madsen KH, Pinborg A, Heede M, Knudsen GM, Siebner HR et al (2015). Role of emotional processing in depressive responses to sex-hormone manipulation: a pharmacological fMRI study. Transl Psychiatry 5: e688.

Hjelmervik H, Hausmann M, Osnes B, Westerhausen R, Specht K (2014). Resting states are resting traits - an fMRI study of sex differences and menstrual cycle effects in resting state cognitive control networks. PLoS ONE 9: e103492.

Jenkinson M, Bannister P, Brady M, Smith S (2002). Improved optimization for the robust and accurate linear registration and motion correction of brain images. Neuroimage 17: 825-841.

Koziol LF, Budding DE, Chidekel D (2011). Sensory integration, sensory processing, and sensory modulation disorders: putative functional neuroanatomic underpinnings. Cerebellum 10: 770-792.

Lisofsky N, Mårtensson J, Eckert A, Lindenberger U, Gallinat J, Kühn S (2015). Hippocampal volume and functional connectivity changes during the female menstrual cycle. Neuroimage 118: $154-162$.

Lundin C, Danielsson KG, Bixo M, Moby L, Bengtsdotter H, Jawad I et al (2017). Combined oral contraceptive use is associated with both improvement and worsening of mood in the different phases of the treatment cycle-a double-blind, placebo-controlled randomized trial. Psychoneuroendocrinology 76: 135-143.

Mahfouz A, Lelieveldt BPF, Grefhorst A, van Weert LTCM, Mol IM, Sips HCM et al (2016). Genome-wide coexpression of steroid receptors in the mouse brain: identifying signaling pathways and functionally coordinated regions. Proc Natl Acad Sci USA 113: 2738-2743.

Menon V. Salience Network. In: Toga AW (ed). Brain Mapping: An Encyclopedic Reference, vol 2. Elsevier/Academic Press: Amsterdam, 2015, pp 597-611.

Montgomery SA, Asberg M (1979). A new depression scale designed to be sensitive to change. Br J Psychiatry 134: 382-389.

Moses EL, Drevets WC, Smith G, Mathis CA, Kalro BN, Butters MA et al (2000). Effects of estradiol and progesterone administration on human serotonin 2A receptor binding: A PET study. Biol Psychiatry 48: 854-860.

Nevatte T, O’Brien PMS, Bäckström T, Brown C, Dennerstein L, Endicott J et al (2013). ISPMD consensus on the management of premenstrual disorders. Arch Womens Ment Health 16: 279-291. 
Nolte J (2009). The Human Brain: An Introduction to its Functional Anatomy. Mosby: Philadelphia, PA.

Olivier B (2015). Serotonin: a never-ending story. Eur J Pharmacol 753: 2-18.

Ottowitz WE, Derro D, Dougherty DD, Lindquist MA, Fischman AJ, Hall JE (2008a). FDG-PET analysis of amygdalar-cortical network covariance during pre- versus post-menopausal estrogen levels: potential relevance to resting state networks, mood, and cognition. Neuroendocrinol Lett 29: 467-474.

Ottowitz WE, Siedlecki KL, Lindquist MA, Dougherty DD, Fischman AJ, Hall JE (2008b). Evaluation of prefrontalhippocampal effective connectivity following 24 hours of estrogen infusion: an FDG-PET study. Psychoneuroendocrinology 33: 1419-1425.

Parsons B, Rainbow TC, MacLusky NJ, McEwen BS (1982). Progestin receptor levels in rat hypothalamic and limbic nuclei. J Neurosci 2: 1446-1452.

Peper JS, Heuvel MP, van den, Mandl RCW, Pol HEH, van Honk J (2011). Sex steroids and connectivity in the human brain: a review of neuroimaging studies. Psychoneuroendocrinology 36: 1101-1113.

Petersen N, Cahill L (2015). Amygdala reactivity to negative stimuli is influenced by oral contraceptive use. Soc Cogn Affect Neurosci 10: $1266-1272$.

Petersen N, Kilpatrick LA, Goharzad A, Cahill L (2014). Oral contraceptive pill use and menstrual cycle phase are associated with altered resting state functional connectivity. Neuroimage 90: 24-32.

Pletzer B, Crone JS, Kronbichler M, Kerschbaum H (2016). Menstrual cycle and hormonal contraceptive-dependent changes in intrinsic connectivity of resting-state brain networks correspond to behavioral changes due to hormonal status. Brain Connect 6: 572-585.

Roy AK, Shehzad Z, Margulies DS, Kelly AMC, Uddin LQ, Gotimer $\mathrm{K}$ et al (2009). Functional connectivity of the human amygdala using resting state fMRI. Neuroimage 45: 614-626.

Sacher J, Rekkas PV, Wilson AA, Houle S, Romano L, Hamidi J et al (2015). Relationship of monoamine oxidase-a distribution volume to postpartum depression and postpartum crying. Neuropsychopharmacology 40: 429-435.

Sang L, Qin W, Liu Y, Han W, Zhang Y, Jiang T et al (2012). Resting-state functional connectivity of the vermal and hemispheric subregions of the cerebellum with both the cerebral cortical networks and subcortical structures. Neuroimage 61: 1213-1225.

Seeley WW, Menon V, Schatzberg AF, Keller J, Glover GH, Kenna $\mathrm{H}$ et al (2007). Dissociable intrinsic connectivity networks for salience processing and executive control. J Neurosci 27: 2349-2356.

Sheehan DV, Lecrubier Y, Sheehan KH, Amorim P, Janavs J, Weiller E et al (1998). The Mini-International Neuropsychiatric Interview (M.I.N.I.): the development and validation of a structured diagnostic psychiatric interview for DSM-IV and ICD-10. J Clin Psychiatry 59: 22-33.

Skovlund C, Mørch L, Kessing L, Lidegaard Ø (2016). Association of hormonal contraception with depression. JAMA Psychiatry 73: 1154-1162.

Sundström Poromaa I, Gingnell M (2014). Menstrual cycle influence on cognitive function and emotion processing-from a reproductive perspective. Front Neurosci 8: 380.

Sundström Poromaa I, Segebladh B (2012). Adverse mood symptoms with oral contraceptives. Acta Obstet Gynecol Scand 91: 420-427.

Tzourio-Mazoyer N, Landeau B, Papathanassiou D, Crivello F, Etard O, Delcroix N et al (2002). Automated anatomical labeling of activations in SPM using a macroscopic anatomical parcellation of the MNI MRI single-subject brain. Neuroimage 15: 273-289.

Vaidya CJ, Gordon EM (2013). Phenotypic variability in restingstate functional connectivity: current status. Brain Connect 3: 99-120.

Yan C-G, Zang Y-F (2010). DPARSF: a MATLAB toolbox for "pipeline" data analysis of resting-state fMRI. Front Syst Neurosci 4: 13.

Zeidan MA, Igoe SA, Linnman C, Vitalo A, Levine JB, Klibanski A et al (2011). Estradiol modulates medial prefrontal cortex and amygdala activity during fear extinction in women and female rats. Biol Psychiatry 70: 920-927.

Zhang Y, Gong J, Xie C, Ye EM, Jin X, Song H et al (2015). Alterations in brain connectivity in three sub-regions of the anterior cingulate cortex in heroin-dependent individuals: evidence from resting state fMRI. Neuroscience 284: 998-1010.

Supplementary Information accompanies the paper on the Neuropsychopharmacology website (http://www.nature.com/npp) 